\title{
Some Qualitative Approach for Bounded Solutions of Some Nonlinear Diffusion Equations with Non-Autonomous Coefficients: Oscillation Criteria
}

\author{
Tadie \\ Mathematics Institut, Universitetsparken 52100 Copenhagen, Denmark $\uparrow$
}

Abstract:

This work investigates some oscillation criteria for the equation

$$
\nabla \cdot\left\{a(x, u) \Phi_{\alpha}(\nabla u)\right\}+c(x, u) \phi_{\alpha}(u)+f(x, u)=0 \quad x \in \Omega \subseteq \mathbb{R}^{n} .
$$

This has been largely done for cases where the coefficients $a$ and $c$ are autonomous (i.e. not depending on the unknown function $u$ ). Using some Picone-type formulas we show that if those coefficients are continuous, positive and bounded away from zero and for small $|t|, \quad 0<\frac{f(x, t)}{\phi_{\alpha}(t)}=O\left(|t|^{\theta}\right)$ with $\theta \geq \alpha>0$, then any bounded and non-trivial solution of the equation is oscillatory.

AMS Subject Classification: $35 \mathrm{~J} 60,35 \mathrm{~J} 70,34 \mathrm{C} 10$

Keywords: Picone's formulae,Nonlinear Diffusion, Oscillation of Solutions, Non-autonomous Coefficients, Quasilinear Elliptic Equations

\subsection{Preliminaries}

\section{Introduction}

Nonlinear elliptic equations arise in many different domains in pure and applied Mathematics. In particular in Nonlinear Diffusion problems,

( slow, fast diffusion flows,...) (see e.g. [1] ). The general equation has the form

$$
-\nabla \cdot\left(K_{1}(x, u, \nabla u)\right)+b(x, u, \nabla u)=0 .
$$

According to the requirements of the given phenomena and characteristics of the problem, this equation can be put into various forms when the coefficients fulfill some specific conditions. Let $\Omega \subset \mathbb{R}^{n}$ be an open domain and $W:=\bar{\Omega} \times \mathbb{R} \times \mathbb{R}^{n}$. In this work, we will be concerned about some models of problems coming from the following general type of problems:

$$
\left\{\begin{array}{l}
\text { (i) } \quad \nabla \cdot\{A(x, u, \nabla u) \nabla u\}+K(x, u, \nabla u) u+f(x, u)=0 ; \quad x \in \Omega \\
\text { where for some } m>0, \quad \forall x \in \bar{\Omega} \text { and } Y \in \mathbb{R}^{n}, \\
\text { (ii) } \quad A \in C^{1}(x, u, Y ;(m, \infty)) ; \quad K \in C\left(\bar{\Omega} \times \mathbb{R} \times \mathbb{R}^{n}, \mathbb{R}\right) ; \\
\text { (iii) } \quad f \in C(\bar{\Omega} \times \mathbb{R}, \mathbb{R}) .
\end{array}\right.
$$

In the sequel a solution of (1.2)(i) will be any $u \in \Re(\Omega)$ which satisfies weakly the equation (i) where

$$
\Re(\Omega):=\left\{w \in C^{1}(\bar{\Omega}) \bigcap C^{2}(\Omega) ; A(x, w, \nabla w) \nabla w \in C^{1}(\bar{\Omega}\} .\right.
$$

Among other, one of the important qualitative aspects of the solutions ( when they are bounded ) of those problems is the oscillation criteria when that solution is obtained (or extended ) in an unbounded domain $\Omega$, say. The oscillatory criteria have been mainly investigated in the literature for the cases where the coefficients $(A(.$.$) and K(.)$.$) are$ autonomous (i.e. independent of the unknown functions $u, \nabla u, .$. ) ( see $[4,6,5,11,12]$ and references therein ). 
A function $u$ is said to be oscillatory in $\Omega$ if $\forall R>0$, it has zeros in

$\Omega_{R}:=\{x \in \Omega ;|x|>R\}$ and strongly oscillatory if its support has non-void open, bounded and connected components in any $\Omega_{R}$.

\subsection{Some recalls on equations with autonomous coefficients}

It is about proiblems whose equations are of the type

$$
\nabla \cdot\left\{A(x) \Phi_{\alpha}(\nabla u)\right\}+C(x) \Phi_{\alpha}(u)+F(x, u, \nabla u)=0 ; \quad x \in \Omega_{R}
$$

where $\alpha>0$ and $\Phi_{\alpha}(S):=|S|^{\alpha-1} S$. For this type of equations, in [6] the theorems 1.5 and 2.4 for one-dimensional cases and 3.1, 3.2 and 3.3 for multidimensional ones offer interesting results when the coefficients $A$ and $C$ are strictly positive functions and some boundedness conditions set on $F$.

In the hypotheses the coefficients $A$ and $C$ are required to keep ( each ) the same sign mainly for technical reasons; the results we establish rely on the fact that the corresponding "half-linear equations"

$$
\nabla \cdot\left\{A(x) \Phi_{\alpha}(\nabla u)\right\}+C(x) \Phi_{\alpha}(u)=0
$$

are odd in the sense that if $u$ solves the equation, so will $-u$. Those results were obtained via usage of some Picone-type formulae and comparison principles.

Results for such problems with $\alpha=1$, namely

$\nabla \cdot\{a(u) \nabla u\}+C(x) u+f(x, u)=0 \quad$ have been investigated in [8].

\section{Models Problems And Main Resultys}

In the sequel we define the following:

$\forall \gamma>0, \quad s \in \mathbb{R}$ and $S \in \mathbb{R}^{n} \quad \phi_{\gamma}(s):=|s|^{\gamma-1} s$ and $\Phi_{\gamma}(S):=|S|^{\gamma-1} S$ and after easy and elaborate calculations, they have the forrlowing properties:

$$
\left\{\begin{array}{l}
s \phi_{\gamma}(s)=|s|^{\gamma+1} ; \quad s \phi_{\gamma}^{\prime}(s)=\gamma \phi_{\gamma}(s) \quad \text { and } \quad \phi_{\gamma}(s t)=\phi_{\gamma}(s) \phi_{\gamma}(t) \\
S \Phi_{\gamma}(S)=|S|^{\gamma+1} \quad \text { and } \quad \Phi_{\gamma}(T S)=\Phi_{\gamma}(T) \Phi_{\gamma}(S) \\
\text { and for a function } s \text { and } S=\nabla s, \quad s \nabla\left[\phi_{\mu}(s)\right]=\mu S \phi_{\mu}(s) .
\end{array}\right.
$$

For the type of equation we will be dealing with, say

$$
\nabla \cdot\left\{a(x, u) \Phi_{\gamma}(\nabla u)\right\}+c(x, u) \phi_{\gamma}(u)+f(x, u)=0 \quad x \in \Omega,
$$

we first lay down the following hypotheses on the coefficients:

For some $\gamma, m>0$

(a): the coefficients $a \in C^{1}(\bar{\Omega} \times \mathbb{R},(m, \infty))$ and

$$
c \in C(\bar{\Omega} \times \mathbb{R},(m, \infty)) \text {; }
$$

(H)

(b): $\quad a$ and $c$ are even in their secong argument

$$
\text { i.e. } \forall(x, t) \in \Omega \times \mathbb{R}, \quad a(x, t)=a(x,-t) \text { and } c(x, t)=c(x,-t) \text {. }
$$

(f): (i) $f \in C(\bar{\Omega} \times \mathbb{R}, \mathbb{R})$ satisfies $\quad \frac{f(x, t)}{\phi_{\alpha}(t)}>0 \quad \forall(x, t) \in \bar{\Omega} \times \mathbb{R} \backslash\{0\}$ and

(ii) $\exists \theta>\alpha ;|f(x, t)|=O\left(|t|^{\theta}\right)$ for small $|t|$. 
The conditions on $f$ mainly the (ii) ensures that for smaff $|u|, \quad \phi_{\alpha}(u)$ remains the leading term ensuring that the solution cannot have a compact support. (see e.g. [1] )

\subsection{Problems without damping terms}

The first model problem is the following:

under thew hypotheses in $(\mathrm{H})$, we consider

for $\Omega \subset \mathbb{R}^{n} ; \quad \alpha, m>0 ; \quad \phi:=\phi_{\alpha}$ and $\Phi:=\Phi_{\alpha}$ the equation

$$
\nabla \cdot\{a(x, u) \Phi(\nabla u)\}+c(x, u) \phi(u)+f(x, u)=0 \quad x \in \Omega .
$$

It is important to note that from (f) above, the function $f$ is a restoring function in $t$ (i.e. $\forall(x, s) \in \Omega \times \mathbb{R} \backslash\{0\}, \quad s f(x, s)>0)$, if $u$ is a solution of the equation (2.2), so would be $-u$; thus we can say that $u$ is non-oscillatory if $u$ is eventually strictly positive or eventually strictly negative, because $a$ and $c$ are set positive.

With $\Omega$ extended to the whole space the following result will be obtained:

Theorem 2.1. Assume that $a, c, f$ satisfy the hypotheses in (H).

Then any non-trivial and bounded solution $u$ of (2.2) in the whole $\mathbb{R}^{n}$ is oscillatory in the sense that it has zeros in any exterior domain $\Omega_{R}$ of $\mathbb{R}^{n}$.

Next we have this slight different result for one-dimensional equations:

Theorem 2.2. Given the functions

$$
\text { (H1) : } \frac{D}{B}, D \in C\left(\mathbb{R}, \mathbb{R}^{+}\right) \text {and the increasing } B \in C^{1}\left(\mathbb{R}, \mathbb{R}^{+}\right) \text {, }
$$

any bounded and non-trivial solution of

$$
\left\{B(u) \phi_{\alpha}\left(u^{\prime}\right)\right\}^{\prime}+D(u) \phi_{\alpha}(u)=0 \quad t \in \Omega_{R} ; \quad u(R)>0
$$

is strongly oscillatory.

\section{Remark 2.2}

As we will see in the proof, the same conclusion holds for the equation

$$
\left\{B(u) \phi_{\alpha}\left(u^{\prime}\right)\right\}^{\prime}+D(u) \phi_{\beta}(u)=0 \quad t \in \Omega_{R} ; \quad u(R)>0
$$

provided that $\beta>0$. (see also $[6,7]$ )

\subsection{Problems with damping terms}

In terms of oscillation character, when the coefficient $a$ satisfies $(H)(a)$, the equation (2.2) and

$$
\nabla \cdot\{\Phi(\nabla u)\}+\frac{\nabla a(x, u)}{a(x, u)} \Phi(\nabla u)+\frac{c(x, u)}{a(x, u)} \phi(u)+\frac{f(x, u)}{a(x, u)}=0
$$

are equivalent in the sense that whenever one oscillates so does the other. For the equation (2.3), $\frac{\nabla a(x, u)}{a(x, u)} \Phi(\nabla u)$ denotes its damping term. Thus for the model of this type of problem we consider in $\Omega$ the problem

$$
\left\{\begin{array}{c}
\text { (i) } \quad \nabla \cdot\{\Phi(\nabla u)\}+B(x, u) \cdot \Phi(\nabla u)+C(x, u) \phi(u)+F(x, u)=0 \\
\text { where } \\
\text { (ii) } \exists b \in C^{1}(\Omega \times \mathbb{R}, \mathbb{R}) \text { such that } \forall u \in C^{1}\left(\mathbb{R}^{n}, R\right) \\
\nabla b(x, u)=B(x, u):=\left.\nabla_{x} b(x, t)\right|_{t-u}+\left.\frac{\partial}{\partial t} b(x, t)\right|_{t-u} \nabla_{x} u \\
\text { (iii) and } C \text { and } F \text { are as } c \text { and } f \text { in }(\mathrm{H}) .
\end{array}\right.
$$

We will establish the following result: 
Theorem 2.3. Under the hypotheses in (2.4)(iii), assume that

a) ether $b(x, t) \equiv b(t) \quad$ and $\forall t \in \mathbb{R} \quad b^{\prime}(t)>0$ or

b) $x \mapsto c(x, t)$ is increasing and unbounded in $|x|$ while for some continuous nonnegative function $\rho \in C(\mathbb{R}), \quad\left|\nabla_{x} b(x, t)\right|<\rho(t) \quad \forall x \in \Omega$.

Then any non-trivial and bounded solution of the equation is oscillatory.

\section{Basic Picone-Type Formulae}

Given two ( supposedly) solutions $u$ and $v$ in $\Omega$ respectively of

$$
\left\{\begin{array}{l}
(\text { a }) \quad \nabla \cdot\{A(x, u, \nabla u) \nabla u\}+K(x, u, \nabla u) u+f(x, u)=0 \quad \text { and } \\
(b) \quad \nabla \cdot\left\{A_{1}(x, v, \nabla v) \nabla v\right\}+K_{1}(x, v, \nabla v) v+f_{1}(x, v)=0,
\end{array}\right.
$$

wherever $v \neq 0$,

$$
\begin{aligned}
& \nabla \cdot\left\{u A(x, u, \nabla u) \nabla u-\frac{u^{2}}{v} A_{1}(x, v, \nabla v) \nabla v\right\}=\left[A-A_{1}\right]|\nabla u|^{2} \\
& +A_{1} Z(u, v)+u^{2}\left[K_{1}-K\right]+u^{2}\left\{\frac{f_{1}}{v}-\frac{f}{u}\right\}
\end{aligned}
$$

where $\forall w, z \in C^{1}$, the nonnegative 2 -form $Z$ is defined by

$$
Z(w, z):=|\nabla w|^{2}-2 \frac{w}{z} \nabla w \nabla z+\left|\frac{w}{z} \nabla z\right|^{2}=\left(\nabla w-\frac{w}{z} \nabla z\right)^{2} .
$$

This type of formula is the main tool for establishing the results. In fact (3.2) is valuable only in a domain where $v$ is non zero. Thus if say $v \neq 0$ in a domain $D$ and $\left.u\right|_{\partial D}=0$ then the integration of (3.2) over $D$ would give 0 at the left.

So if it happens that the right hand of (3.2) is strictly positive ( or negative ) in that $D$, we have a contradiction implying that $v$ has zero inside that $D$.

\section{Equations Without Damping Terms}

Given a bounded region $\Omega \subset \mathbb{R}^{n}$, an $\alpha, m>0$, and

$$
\text { in the sequel, unless indicated otherwise } \phi(\Phi):=\phi_{\alpha}\left(\Phi_{\alpha}\right) \text {, }
$$

we consider the equation (2.2) under the hypotheses $(\mathrm{H})$.

AS seen in (3.2)-(3.3), if $v, w$ are solutions for

$$
\nabla \cdot\left\{a_{1}(x, v) \Phi(\nabla v)\right\}+c_{1}(x, v) \phi(v)+f_{1}(x, v)=0 \text { and }
$$

$\nabla \cdot\{a(x, u) \Phi(\nabla u)\}+c(x, u) \phi(u)+f(x, u)=0$ respectively,

formally wherever $v \neq 0$

$$
\left\{\begin{array}{c}
\nabla \cdot\left\{u a(x, u) \Phi(\nabla u)-u \phi\left(\frac{u}{v}\right) a_{1}(x, v) \Phi(\nabla v)\right\}= \\
{\left[a(x, u)-a_{1}(x, v)\right]|\nabla u|^{\alpha+1}+a_{1}(x, v) \zeta_{\alpha}(u, v)+} \\
u^{\alpha+1}\left[c_{1}(x, v)-c(x, u)\right]+u^{\alpha+1}\left\{\frac{f_{1}(x, v)}{\phi(v)}-\frac{f(x, u)}{\phi(u)}\right\}
\end{array}\right.
$$


where $\forall z, y \in C^{1}(\Omega)$ the non-negative two-form $\zeta_{\gamma},(\gamma>0)$ is defined by

$$
\zeta_{\gamma}(z, y):=|\nabla z|^{\gamma+1}-(\gamma+1)\left|\frac{z}{y} \nabla y\right|^{\gamma-1} \frac{z}{y} \nabla y \cdot \nabla z+\gamma\left|\frac{z}{y} \nabla y\right|^{\gamma+1}
$$

which is positive and is zero only if $\exists \mu \in \mathbb{R} ; \quad z=\mu y$ ([11, 12]).

These types of equations are found in various Reaction Diffusion problems like flows through porous media, Plasma Physics ([11,9]).

Unless indicated otherwise, in the sequel the coefficients $a_{i}(x,$.$) in the principal parts$ of the equations and $c_{i}(x,$.$) of the half-linear part will fullfil the hypotheses in (\mathrm{H})$.

\subsection{Proof of Theorem 2.1}

Proof. Let $u$ be such a bounded solution and $b:=|u|_{\infty}$. It is known that for $A(x):=$ $\max _{|t| \leq b}\{a(x, t)\}$

$$
\nabla \cdot\{A(x) \Phi(\nabla w)\}+m \phi(w)=0
$$

is oscillatory given the hypotheses on $a(.,$.$) ( see [6] ).$

Assume that $u>0$ in some $\Omega_{R}:=\left\{x \in \mathbb{R}^{n} ; \quad|x|>R\right\}$. With this $w,(4.1)$ reads in $\Omega_{R}$

$$
\begin{aligned}
& \nabla \cdot\left\{w A(x) \Phi(\nabla w)-w \phi\left(\frac{w}{u}\right) a(x, u) \Phi(\nabla u)\right\}=[A(x)-a(x, u)]|\nabla w|^{\alpha+1} \\
& +a(x, u) \zeta_{\alpha}(w, u)+w^{\alpha+1}[c(x, u)-m]+w^{\alpha+1} \frac{f(x, u)}{\phi(u)}
\end{aligned}
$$

whose integration over any nodal set $D(w) \subset \Omega_{R}$ would give

$$
\begin{aligned}
& 0=f_{D(w)}\left\{[A(x)-a(x, u)]|\nabla w|^{\alpha+1}+\right. \\
& \left.a(x, u) \zeta_{\alpha}(w, u)+w^{\alpha+1}[c(x, u)-m]+w^{\alpha+1} \frac{f(x, u)}{\phi(u)}\right\} d x>0 \\
& \text { which is absurd if } u>0 \text { in } \Omega_{R} \text {. Therefore } u>0 \text { in } \Omega_{R} \text { cannot }
\end{aligned}
$$

which is absurd if $u>0$ in $\Omega_{R}$. Therefore $u>0$ in $\Omega_{R}$ cannot hold for any $R>0$,

\subsection{Proof of Theorem 2.2}

Proof. Althnough this result can be an application of Theorem 2.1, we provide here an analytical proof.

With

$$
\text { increasing } B \in C^{1}\left(\mathbb{R}, \mathbb{R}^{+}\right) \text {and } \frac{D}{B}, D \in C\left(\mathbb{R}, \mathbb{R}^{+}\right) \text {, }
$$

if for some $R>0$ a bounded and non-trivial solution $u$ of

$$
\left\{B(u) \phi_{\alpha}\left(u^{\prime}\right)\right\}^{\prime}+D(u) \phi_{\alpha}(u)=0 \quad t \in \Omega_{R^{*}} \quad u(R)>0
$$

is strictly positive and bounded then from the equation

$$
\begin{aligned}
& u^{\prime}(t) B^{\prime}(u) \phi_{\alpha}\left(u^{\prime}\right)+B(u)\left[\phi_{\alpha}\left(u^{\prime}\right)\right]^{\prime}=B^{\prime}(u)\left|u^{\prime}\right|^{\alpha+1}+B(u)\left[\phi_{\alpha}\left(u^{\prime}\right)\right]^{\prime} \\
& =-D(u) \phi_{\alpha}(u) \leq 0 \text { in } \Omega_{R} .
\end{aligned}
$$

This implies that $\left[\phi_{\alpha}\left(u^{\prime}\right)\right]^{\prime}=u^{\prime \prime} \phi_{\alpha}^{\prime}\left(u^{\prime}\right)=\alpha\left|u^{\prime}\right|^{\alpha-1} u^{\prime \prime} \leq 0$. So, $u^{\prime \prime} \leq 0$ in $\Omega_{R}$ and being bounded, $u^{\prime} \geq 0$ and decreases to zero there.

Thus if $u(t)>0 \quad \forall t>R$, it increases to its upper bound in $\Omega_{R}$. Let $M:=\sup _{\Omega_{R}} u(t)$ and $\delta:=\min _{[u(T), M]} \frac{D(u)}{B(u)}$. From the equation

$\phi_{\alpha}\left(u^{\prime}(t)\right) \leq \phi_{\alpha}\left(u^{\prime}(T)\right)-\delta M(t-T), \quad \forall t>T$ which is eventually strctly negative, conflicting with the fact that $u^{\prime} \geq 0$ in $\Omega_{T}$. Therefore no bounded and non-trivial solution of the equation can be eventually positive. 
Let

\section{Equations With Damping Terms}

$$
\left\{\begin{array}{l}
b \in C^{1}\left(\mathbb{R}^{n} \times \mathbb{R},(0, \infty)\right) ; \quad B(x, t):=\nabla b(x, t) ; \\
c(x, t) \text { and } f(x, t) \text { are as those in }(\mathrm{H}) .
\end{array}\right.
$$

Let for some strictly positive $C \in C(\mathbb{R},(0, \infty) \quad u$ and $v$ be respectively solutions of

$$
\begin{cases}(\text { i }) & \nabla \cdot\{\Phi(\nabla u)\}+B(x, u) \cdot \Phi(\nabla u)+c(x, u) \phi(u)+f(x, u)=0 ; \\ (\text { ii }) & \nabla \cdot\{\Phi(\nabla v)\}+m \phi(v)=0 .\end{cases}
$$

Assume that $u \neq 0$ in say, $D \subset \mathbb{R}^{n}$. Then in $D$ easy calculations show that with $\Gamma(v, u):=v \Phi(\nabla v)-v \Phi\left(\frac{v}{u} \nabla u\right)$,

the corresponding Picone-type formulae are:

$$
\left\{\begin{array}{c}
\nabla \cdot\{b(x, u) \Gamma(v, u)\}=b(x, u)\left\{\zeta_{\alpha}(v, u)+\right. \\
\left.\left[c(x, u)-m+\frac{f(x, u)}{\phi(u)}\right]|v|^{\alpha+1}+v B(x, u) \cdot \Phi\left(\frac{v}{u} \nabla u\right)\right\} \\
+B(x, u) \cdot \Gamma(v, u) .
\end{array}\right.
$$

\subsection{Proof of Theorem 2.3}

\section{Proof. .}

We chose as before $m:=\min _{|t| \leq b}\{c(x, t)\}$.

Assume that there is such a solution $u$ for (2.4)(i) such that $|u|_{\infty}=b$.

$$
\nabla \cdot\{\Phi(\nabla v)\}+m \phi(v)=0
$$

is oscillatory and as in (5.3)(a), a version of Picone-type formula for $v$ and $u$ reads

$$
\begin{aligned}
& \nabla \cdot\{b(u) \Gamma(v, u)\}=B(u) \cdot \Gamma(v, u)+b(u)\left\{\zeta_{\alpha}(v, u)+\right. \\
& \left.\left[c(x, \beta)-m+\frac{f(x, u)}{\phi(u)}\right]|v|^{\alpha+1}+v B(x, u) \cdot \Phi\left(\frac{v}{u} \nabla u\right)\right\} .
\end{aligned}
$$

a) The equation (5.2)(i) is unchanged if $b$ is replaced by $b(u)+\lambda, \quad \forall \lambda \in \mathbb{R}$. If we assume that $u>0$ in some $\Omega_{R}$, then for any such $\lambda$, the integration of (5.4) over any nodal set $D\left(v^{+}\right) \subset \Omega_{R}$ gives

$$
\begin{aligned}
& 0=\int_{D\left(v^{+}\right)}(b(u)+\lambda)\left\{\zeta_{\alpha}(v, u)+\left[c(x, \beta)-m+\frac{f(x, u)}{\phi(u)}\right]|v|^{\alpha+1}\right. \\
& \left.+v B(x, u) \cdot \Phi\left(\frac{v}{u} \nabla u\right)\right\} d x+\int_{D\left(v^{+}\right)} B(x, u) \cdot \Gamma(v, u) d x .
\end{aligned}
$$

For this to hold for any arbitrary $\lambda$, each integrand has to be zero in $D\left(v^{+}\right)$and in particular

$$
\zeta_{\alpha}(v, u)+\left[c(x, \beta)-m+\frac{f(x, u)}{\phi(u)}\right]|v|^{\alpha+1}+v B(x, u) \cdot \Phi\left(\frac{v}{u} \nabla u\right) \equiv 0 .
$$


But $v B(x, u) \cdot \Phi\left(\frac{v}{u} \nabla u\right)=v b^{\prime}(u)|\nabla u|^{\alpha-1}|\nabla u|^{\alpha+1} \geq 0$ and each term in the equation above is non-negative. Therefore the assumption that such a bounded non-trivial solution $u$ can remain strictly positive eventually is not possible.

b) In this case, the segment $\left[c(x, \beta)-m+\frac{f(x, u)}{\phi(u)}\right]|v|^{\alpha+1}+v B(x, u) \cdot \Phi\left(\frac{v}{u} \nabla u\right)$ remains strictly positive eventually as $c(x, t)$ is unbounded and

$\left|v B(x, u) \cdot \Phi\left(\frac{v}{u} \nabla u\right)\right| \leq$ const. $|v|^{\alpha+1}$.

Therefore that segment is eventually positive.

\section{Some Applications}

Oscillation criteria for a porous medium equation with source (see [3] )

Such an equation (in steady form ) can be

$$
\left\{\begin{array}{c}
\nabla \cdot\left\{\Phi_{\alpha}(\nabla V(x))\right\}+\phi_{p}(u)=0 \quad x \in \mathbb{R}^{n} ; n>1 ; \\
\beta, \alpha, p>0 ; \quad \text { with } V(x):=\phi_{\beta}(u)=|u(x)|^{\beta-1} u(x) .
\end{array}\right.
$$

As $\nabla \cdot\left\{|u(x)|^{\beta-1} u(x)\right\}:=\nabla \cdot\left\{\left(u(x)^{2}\right)^{[\beta-1] / 2} u(x)\right\}, \nabla \cdot \Phi_{\alpha}(\nabla V(x))=\beta^{\alpha}|u(x)|^{\alpha(\beta-1)} \Phi_{\alpha}(\nabla u)$ and the equation in (6.1) becomes

$$
\left\{\begin{array}{l}
\text { (i) } \quad \nabla \cdot\left\{|u(x)|^{\alpha(\beta-1)} \Phi_{\alpha}(\nabla u)\right\}+\beta^{-\alpha} \phi_{p}(u)=0 ; \quad x \in \mathbb{R}^{n} ; \\
\text { and wherever } u \neq 0, \text { it becomes for } F(u, \nabla u):=\frac{\alpha(\beta-1) u}{|u|^{2}}|\nabla u|^{\alpha+1} \\
\text { (ii) } \quad \nabla \cdot\left\{\Phi_{\alpha}(\nabla u)\right\}+F(u, \nabla u)+\beta^{-\alpha} \phi_{p}(u)=0 ; \quad x \in \mathbb{R}^{n} .
\end{array}\right.
$$

\section{One-dimensional problem}

In one-dimension, for $V(t)=|u(t)|^{\beta-1} u(t)$, after some calculations

$$
\left\{|u|^{(\beta-1) \alpha} \phi_{\alpha}\left(u^{\prime}\right)\right\}^{\prime}+\phi_{p}(u)=0, t>0 .
$$

Wherever $u \neq 0$, this equation is equivalent to

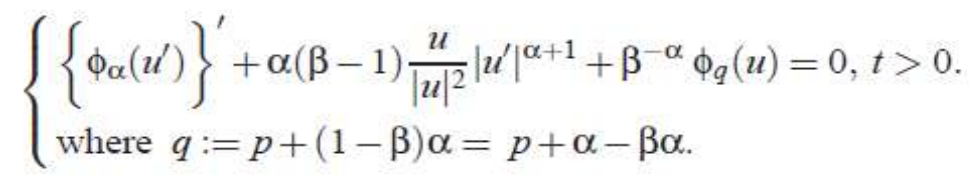

Theorem 6.1. Let

$\alpha>0, \beta>1$ and $p+\alpha>\alpha \beta$.

Then any bounded and non-trivial solution of (6.4) is oscillatory i.e. (6.3) is oscillatory.

Proof. Assume that there is a bounded and non-trivial solution $u \in C^{2}([0, \infty))$ such that $u(r)>0$ in some $\Omega_{R}, R>0$.

When $p+\alpha>\alpha \beta, \quad q>0$. In $\Omega_{R}$ the middle term of (6.4) is positive and the result is an application of Theorem 2.4 of [6]. 


\section{Multidimensional problem}

From Theorem 3.3 of [6] we have

Theorem 6.2. If $q=\alpha$ or $p=\alpha \beta$ then (6.2)(ii) is oscillatory as $u F(u, \nabla u)>0 \quad \forall u \neq 0$.

\subsection{Radially symmetric solutions}

If there is a radially symmetric solution with $V(r):=\phi_{\beta}(u(|x|))=|u|^{\beta-1} u ; r:=|x|$ of the equation, then easy calculations give

and for ease writing we consider

$$
\left\{r^{n-1}|u(r)|^{\alpha(\beta-1)} \phi_{\alpha}\left(u^{\prime}\right)\right\}^{\prime}+\frac{r^{n-1}}{\beta^{\alpha}} \phi_{p}(u)=0, \quad r>0
$$

$$
\left\{r^{n-1}|u(r)|^{\alpha(\beta-1)} \phi_{\alpha}\left(u^{\prime}\right)\right\}^{\prime}+r^{n-1} \phi_{p}(u)=0, \quad r>0 .
$$

If we assume that $u>0$ in say, $\Omega_{R}$ then there this equation in $u$ becomes

$$
\left\{\begin{aligned}
& \text { (i) } \quad\left\{r^{n-1} \phi_{\alpha}\left(u^{\prime}\right)\right\}^{\prime}+\frac{\alpha(\beta-1) r^{n-1}}{u}\left|u^{\prime}\right|^{\alpha+1} \\
&+r^{n-1}|u|^{(1-\beta) \alpha} \phi_{p}(u)=0 \text { or } \\
& \text { (ii) } \quad\left\{r^{n-1} \phi_{\alpha}\left(u^{\prime}\right)\right\}^{\prime}+\frac{\alpha(\beta-1) r^{n-1}}{u}\left|u^{\prime}\right|^{\alpha+1}+r^{n-1} \phi_{q}(u)=0
\end{aligned}\right.
$$

with

$$
q=p+\alpha-\alpha \beta \text {. Because } u>0 \text { in } \Omega_{T} \Longrightarrow \frac{\alpha(\beta-1) r^{n-1}}{u}\left|u^{\prime}\right|^{\alpha+1} \geq 0 \text { there, (6.6) is }
$$
oscillatory if

$$
\left\{r^{n-1} \phi_{\alpha}\left(u^{\prime}\right)\right\}^{\prime}+r^{n-1} \phi_{q}(u)=0 ; \quad u(0)=0 ; u^{\prime}(R)=b>0
$$

is oscillatory. (see [6])

We recall also that similarly $(6.7)$ is oscillatory in $\Omega_{R} ; R>0$ if $\left\{\phi_{\alpha}\left(u^{\prime}\right)\right\}^{\prime}+\frac{n-1}{r} \phi_{\alpha}\left(u^{\prime}\right)+\phi_{q}(u)=0 ; \quad r>R$ is.

As the term $\frac{n-1}{r} \phi_{\alpha}\left(u^{\prime}\right)$ has the form $\left[\log \left(r^{n-1}\right)\right]^{\prime} \phi_{\alpha}\left(u^{\prime}\right)$ (damping term), (6.7) will be oscillatory if ( as $q=\alpha+(p-\alpha \beta)$ )

$$
\left\{\phi_{\alpha}\left(u^{\prime}\right)\right\}^{\prime}+|u|^{p-\alpha \beta} \phi_{\alpha}(u)=0 ; \quad r>R \quad \text { is. }
$$

From [2] if $u \in C^{2}(\Omega)$ is a non negative solution then a Pohozaev formula for $u$ reads

$$
\left\{\begin{array}{c}
R^{n}\left\{\frac{\alpha}{\alpha+1}\left|u^{\prime}(R)\right|^{\alpha+1}+\frac{u(R)^{q+1}}{q+1}+\frac{n-(\alpha+1)}{(\alpha+1) R} \phi_{\alpha}\left(u^{\prime}(R)\right) u(R)\right\} \\
=\left[\frac{n(\alpha-q)}{(q+1)(\alpha+1)}+1\right] \int_{0}^{R} r^{n-1} u(r)^{q+1} d r, \quad \forall R>0 .
\end{array}\right.
$$

Define $\Gamma(\alpha, \beta, p):=\left[\frac{n(\alpha-q)}{(q+1)(\alpha+1)}+1\right]=\frac{n(\alpha \beta-p)}{(\alpha+1)(p+\alpha-\alpha \beta+1)}+1$

As $u(0)=0$ and $u^{\prime}(0)>0, u(r)>0$ in some $(0, \mu)$.

If $\exists R_{1}:=\min \left\{R>0 \mid u^{\prime}(R)=0\right\}$, then

$$
\frac{R_{1}^{n} u\left(R_{1}\right)^{q+1}}{q+1}=\Gamma(\alpha, \beta, p) \int_{0}^{R_{1}} r^{n-1} u(r)^{q+1} d r
$$


and obviously such an $R_{1}$ would not exist if $\Gamma(\alpha, \beta, p)<0$. Thus any chance of finding an oscillatory solution for (6.7) requires that $\Gamma(\alpha, \beta, p)>0$ i.e.

$$
\left\{\begin{array}{l}
\text { if }(6.7) \text { is oscillatory then } \\
\Gamma(\alpha, \beta, p):=\left[\frac{n(\alpha-q)}{(q+1)(\alpha+1)}+1\right]=\frac{n(\alpha \beta-p)}{(\alpha+1)(p+\alpha-\alpha \beta+1)}+1>0 .
\end{array}\right.
$$

Notice that if $q \leq \alpha$ then this necessary conditition holds (as in Theorem 6.2 above).

We finally have the following result for the radially symmetric equation (6.5), as applications of results in [6]:

Theorem 6.3. Given $\alpha>0$ and $\beta>1$, if ether

a) $p=\alpha \beta$ or

b) $0 \leq p-\alpha \beta<\frac{(p+\alpha+1-\alpha \beta)(\alpha+1)}{n}$ or

c) $0<p<\alpha \beta$,

then any non-trivial and bounded solution for (6.5) is strongly oscillatory.

Proof. First each of the conditions a)-c) implies that the necessary condition (6.10) holds.

The theorem 1.5 of [6] applies to (6.7) if a) holds:

the Theorem 1.3 of [6] applies to (6.8) if b) holds and the theorem 1.4 of [6] applies to (6.8) if c) holds.

\section{Dedications}

To my late brother Fopa Kayem Rigobert and grand-son Kuate Ceddrick:

Que nos ancêtres vous guident vers la sérénité éternelle.

\section{References}

[1]. J.I.Diaz; Nonlinear Differential equations and Free Boundaries. London, Pitman,1985.

[2]. N.Kawano, WM. Ni and S. Yotsutani; A generalized Pohozaev identity and its application, J.Math. Soc. Japan Vol.42,No3,1990 pp.541-564.

[3]. Claus Dohman ; Pohozaev-Type Inequality for a Quasilinear Haraux-Weissler Equation, Funkcialaj Ekvacioj 39 (1996) $389-397$.

[4]. Tadi'e; Comparison Results for Quasilinear Elliptic equations via Picone-type Identity: Part II: cases where he coefficient of the principal part depends on he unknown function J. Nonlinear Anal., T.M.A.,vol. 71(2009)e601-e606 .

[5]. Tadi'e ; Oscillation criteria for damped Quasilinear second-order Elliptic Equations, Electronic J. of Differential Equations vol. 2011(2011), No.151, pp.1-11.

[6]. Tadi'e ; ON STRONG OSCILLATION CRITERIA FOR BOUNDED SOLUTIONS FOR SOME QUASILINEAR SECOND ORDER ELLIPTIC EQUATIONS , Communications in Mathematical Analysis, volume 13, Number 2, pp. 15-26 (2012) ISSN 1938-9787

[7]. Tadi'e ; Oscllation Criteria for Some Emden-Fowler Type Half-linear Elliptic Equations, Int. Journal of Math. Analysis, Vol.8, 2014, No.24 1165-1168. ( HIKARI Ltd, WWW.m-hikari.com)

[8]. Tadi'e ; OSCILLATION CRITERIA FOR BOUNDED SOLUTIONS FOR SOME NONLINEAR DIFFUSION EQUATIONS VIA PICONE-TYPE FORMULAE, Communications in Mathematical Analysis, volume 12, Number 2, pp. 1-10 (2012) ISSN 1938-9787

[9]. Tian Ma \& Shouhong Wang; Bifurcation Theory and Applications. World Scientific Publ.Co (June 2005). London, Pitman,1985.

[10]. Ravi P. Agarwal, Said R.Grace and Donal O'Regan; Oscillation Theory for Second Order Linear , Half-linear, Superlinear and Sublinear Dynamic Equations. Kluwer, 2000.

[11]. J. Jaros, T. Kusano , N. Yosida; Picone-type Inequalities for Nonlinear Elliptic Equations and their Applications J. of Inequal. \& Appl. (2001), vol. 6, 387-404

[12]. T. Kusano, J. Jaros, N. Yoshida; A Picone-type identity and Sturmian comparison and oscillation theorems for a class of half-linear partial differential equations of second order, Nonlinear Analysis, Vol. 40 (2000), 381-395.

[13]. NorioYoshida ; Forced oscillation criteria for superlinear-sublinear elliptic equations via picone-type inequality , J. Math. Anal. Appl. 363(2010), 711-717. 